\title{
Oral Health-related Quality of Life in Patients with Dystrophic Epidermolysis Bullosa
}

Giulio Fortuna ${ }^{1,2,3 *}$, Massimo Aria4, Sarah Whitmire ${ }^{5}$, Rodrigo Cepeda-Valdes ${ }^{2}$, Sandra Cecilia GarciaGarcia루 Maria Guadalupe Moreno-Trevino $^{6}$, and Julio Cesar Salas-Alanís ${ }^{2,6}$

Received: 09 August 2016; Returned for revision: 01 September 2016; Received in revised form: 05 September 2016; Accepted: 10 September 2016; Published online: 1 October 2016

(C) The author(s) 2016. Published with open access at www.uscip.us

\section{Abstract}

Background: Dystrophic epidermolysis bullosa (DEB) is a devastating mucocutaneous inherited disorder that can have a remarkable impact on the oral cavity.

Objective: To understand the relationships between oral health-related quality of life and general quality of life in DEB patients versus a control group.

Methods: Twenty-eight DEB patients and 26 healthy individuals completed the following battery of scales: Oral Health Impact Profile (OHIP-49), the RAND Short Form-36 (SF-36), the Hamilton Rating Scale for anxiety (HAM-A) and depression (HAM-D).

Results: All 7 dimensions in OHIP-49 demonstrated a statistically significant difference in functional limitations (Median:13.0; IQR:10.3-20.0 vs Median:7.0; IQR:2.0-9.0; $\mathrm{p}<0.001$ ) and physical disability (Median:11.5; IQR:4.0-15.0 vs Median:0.0; IQR:0.0-2.3; p<0.001) in DEB patients vs controls, respectively. In RAND SF-36, physical functioning and general health were highly significant $(\mathrm{p}<0.001)$, whereas limitation due to physical health $(\mathrm{p}=0.043)$ and pain $(\mathrm{p}=0.010)$ were moderately significant. A positive correlation was found between oral health-related quality of life and anxiety ( $p=0.005$ and $p=0.03$ in DEB patients and controls, respectively) and depression ( $\mathrm{p}=0.045$ and $\mathrm{p}=0.001$ in DEB patients and controls, respectively). In

*Corresponding e-mail: giulio.fortuna@gmail.com

1 Department of Diagnostic Science, Louisiana State University School of Dentistry, 1100 Florida Ave, New Orleans, LA, 70124, USA.

2 D.eb.RA. Mexico Foundation, Otomí \#211, casi esq. P. Elías Calles Colonia Azteca, Guadalupe N.L., 67150, Monterrey, Nuevo Leon, Mexico.

3 Federico Navarro Institute - School of Orgonomy "Piero Borrelli", Corso Umberto I, 35, 80138, Naples, Italy.

4 Department of Economics and Statistics, Federico II University of Naples, via Cinthia, Monte Sant'Angelo, 80126, Naples, Italy.

5 Department of Bioinformatics and Genomics, University of North Carolina, 9201 University City Blvd, Charlotte, NC, 28223, USA

6 Department of Basic Science, Universidad de Monterrey, Av. Ignacio Morones Prieto 4500 Pte., 66238 San Pedro Garza García, N.L. México 
DEB patients, no correlation was found between general quality of life and anxiety and depression, oral health-related quality of life and oral-pharyngeal severity score, number of oral-pharyngeal sites involved and general quality of life, as well as oral health-related quality of life between patients with dominant and recessive DEB forms ( $p>0.05)$.

Conclusions: The oral health-related quality of life is highly impaired in patients with DEB. General quality of life was affected only for some dimensions and was not significantly correlated with oral health-related quality of life.

Keywords: Epidermolysis bullosa, OHIP-49, oral health-related quality of life, quality of life, SF-36, anxiety, depression

\section{Introduction}

Epidermolysis bullosa (EB) is a rare, genetic disease characterized by extreme fragility of the skin and mucous membranes, resulting in blisters and/or erosion formation following minimal mechanical trauma (Laimer M, 2015). EB has been classified into four major types: EB simplex (EBS), Junctional EB (JEB), Dystrophic EB (DEB), and mixed (Kindler syndrome). In addition, more than 30 clinical subtypes have been defined (Fine et al., 2014).

EB is a multi-systemic disease, involving not just the skin, but mucous membranes and multiple organs (Fine et al., 2009a; Fine et al., 2009b). EB patients commonly experience frequent serous and/or haemorrhagic blisters and/or erosions, erythema, and atrophy in the oral-pharyngeal cavity, due to its soft tissue fragility (Fortuna et al., 2015a; Fortuna et al., 2013a; Wright, 2010) (Figure 1). This is particular prominent in EB patients carrying the dystrophic type, in which the eroded oral-pharyngeal areas ultimately tend to heal with a scarring phenotype and, rarely, oral milia (Fortuna et al., 2013a; Serrano-Martinez et al., 2003; De Benedittis et al., 2004; Azrak et al., 2006; Wright, 1991; Wright, 1993a; Wright, 1993b). However, in DEB the oral-pharyngeal phenotype does not seem to correlate with the type of genetic mutation (Fortuna et al., 2014).

Several studies have demonstrated that in EB many activities may have a profound impact on general quality of life (Horn et al., 2002; Tabolli et al., 2009; Jeon et al., 2016; Angelis et al., 2016), including eating, drinking, chewing, talking, swallowing, or brushing teeth. However, no data are currently available on the impact of those activities on the oral-pharyngeal cavity.

The aim of this cross-sectional study was to analyze the oral health-related quality of life in patients with DEB versus a control group, and to determine whether there was a correlation between oral health-related quality of life with general quality of life and anxiety and depressive symptoms. 
Giulio Fortuna, Massimo Aria, Sarah Whitmire et al. / American Journal of Oral Medicine

(2016) Vol. 2 No. 2 pp. 43-59
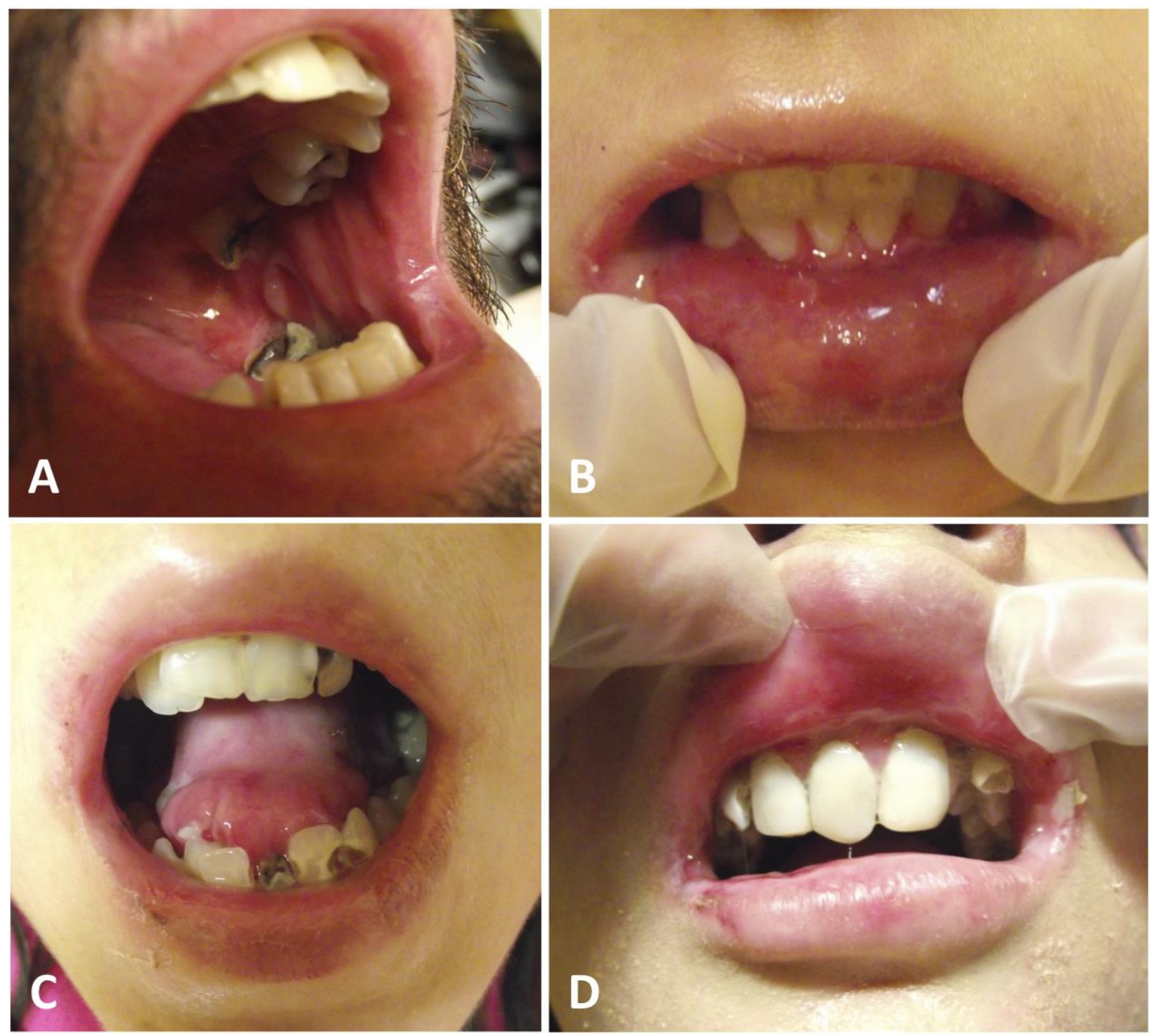

Fig. 1. Oral lesions in patients with Dystrophic Epidermolysis bullosa

A) Erythematous and erosive lesions of the left buccal mucosa; B) Diffuse erythematous lesions and presence of blisters of the lower labial mucosa; C) Presence of microstomia, accompanied by atrophic and bullous/erosive lesions of the tongue; D) Presence of microstomia, accompanied by marked erythema of the upper labial mucosa, bullous/erosive lesions of the upper gingival fornix, and of the commissural areas bilaterally.

\section{Materials and Methods}

\subsection{Study design and Patients}

We designed a cross-sectional study enrolling 28 DEB patients coming from Universidad de 
Monterrey and the EB Clinic at D.eb.RA. Mexico, Monterrey (Mexico), and 26 healthy individuals, as a control group, matched for age and gender. The control group consisted of individuals who work at D.eb.RA. Mexico as volunteer or caregivers, who were consulted exclusively for dental disease, such as dental caries or periodontal disease. All patients provided their written informed consent. This study was approved by the Universidad de Monterrey (Mexico). All DEB and control patients met specific inclusion and exclusion criteria as previously published (Fortuna et al., 2016).

At the time of admission, two specialists (R.C-V., C.G-G.) were responsible for selecting DEB patients and healthy individuals, and collecting all demographic and medical data for both groups. Two other specialists (G.F., J.C.S-A) were responsible for a complete medical and dental anamnesis (chief complaint (if any), history of present illness (if any), past medical history, family, social history and dental history), a general medical examination, and an intra and extraoral examination.

After collecting all these data, DEB patients and controls were asked to respond to the following questionnaires: the Oral Health Impact Profile (OHIP-49); the RAND Short Form-36 (SF-36), the Hamilton Rating Scale for Depression (HAM-D), the Hamilton Rating Scale for Anxiety (HAM-A). The Spanish versions of these scales have been validated and were administrated to each individual in both groups (Castrejón-Pérez et al., 2010; Alonso et al., 1995; Lobo et al., 2002; Ramos-Brieva et al., 1988).

The epidermolysis bullosa oropharyngeal severity score (EBOS) (Fortuna et al., 2013b; Fortuna et al., 2015b), was used to calculate a possible correlation between the objective oral severity score with the oral health-related quality of life

2.2 Psychometric instruments: evaluation of oral health-related quality of life, general quality of life, anxiety and depression.

\subsubsection{The Oral Health Impact Profile (OHIP-49) questionnaire.}

The validated Spanish version of this instrument explores patient perceived quality of life related to oral health based on 49 questions divided into three major dimensions (physical, psychological, and social), and 7 sub-dimensions. Each question is answered using a Likert-type scale scored from 0-4 points as follows: 0 = never, 1 = hardly ever, $2=$ occasionally, 3 = fairly often, $4=$ very often (LopezJornet et al., 2010). The 7 sub-dimensions score ranges are as follows: Functional limitation (9 items): range 0-36; Physical pain (9 items): range 0-36; Psychological discomfort (5 items): range 0-20; Physical disability ( 9 items): range 0-36; Psychological disability (6 items): range 0-24; Social disability (5 items): range 0-20; and Disability (6 items): range 0-24. The OHIP-49, in contrast to other quality of life questionnaires, yields a final summarizing score (including all the items), reflecting better or worse patient perception of oral quality of life: 49 (items) x 0-4 (possible range of the Likert-type scale) $=0-196$. In this model, the higher the scores, the poorer state of oral health (Lopez-Jornet et al., 2010).

\subsubsection{The RAND Short Form-36}

The RAND SF-36 includes 36 items in a Likert-type or forced-choice format, intended to measure the following two major dimensions: physical and mental health. The eight sub-dimensions are as follows: Physical functioning, Role functioning/physical, Role functioning/emotional, 
Giulio Fortuna, Massimo Aria, Sarah Whitmire et al. / American Journal of Oral Medicine

(2016) Vol. 2 No. 2 pp. 43-59

Energy/fatigue, Emotional well-being, Social functioning, Pain, and General health. Scores for each domain range from 0 to 100 , with higher scores indicating a better general quality of life (Arriens et al., 2015).

\subsubsection{The Hamilton rating scale for anxiety (HAM-A)}

This scale consists of 14 items, each defined by a series of symptoms, used to determine patient anxiety levels and distribution of symptoms. Each cluster of symptoms is rated on a five-point scale: $0=$ none, $1=$ mild, $2=$ moderate intensity, $3=$ severe, and $4=$ very severe or grossly disabling. The questionnaire yields a total score range of $0-56$, with higher scores indicating higher levels of anxiety (Hamilton, 1959). Results are interpreted as follows: scores $\leq 7$ indicate an absence/minimal anxiety, scores of 8 to 14 indicate mild, scores 15 to 23 indicate moderate depression, and scores of $\geq 24$ indicate severe anxiety (Matza et al., 2010).

\subsubsection{The Hamilton Rating Scale for Depression (HAM-D)}

The HAM-D contains 21 ratings measured on three ( 0 to 2 ) or five ( 0 to 4 ) point scales. The first 17 items are used in scoring the instrument. Eight items $(4,5,6,12,13,14,16,17)$ that are difficult to quantify are rated on a 0 to 2 scale: $0=$ symptom absent, 1 =slight or doubtful, and $2=$ clearly present. Nine items $(1,2,3,7,8,9,10,11,15)$ are graded more finely on a 0 to 4 scale in terms of increasing intensity: $0=$ symptom absent, $1=$ doubtful or trivial, $2=$ mild, $3=$ moderate, and $4=$ severe. Scores can range from 0 to 52 (Hamilton, 1960). A total score is computed to reflect the degree of symptom severity. Hamilton did not specify cutting-points, but it is generally agreed that scores lower than 7 indicate an absence of depression, scores of 8 to 16 indicate mild depression, scores 17 to 23 indicate moderate depression, and scores $\geq 24$ indicate severe depression (Zimmerman et al., 2013).

\subsection{Evaluation of oral lesions.}

\subsubsection{The Epidermolysis bullosa oropharyngeal severity score (EBOS)}

The EBOS is a validate instrument to assess the oropharyngeal disease severity, made up of 2 components: the disease activity score evaluated on each of the 13 different anatomic sites affected by one or more clinical signs (erythema, atrophy, blister, erosion/ulcer). 1 point is assigned to each clinical sign present in each anatomical site, leading to a total score ranging from 0 to 52 . The other component is the structural damage score that evaluates the presence or absence of the 4 structural damages (microstomia, ankyloglossia, intraoral scars beyond microstomia and ankyloglossia, enamel hypoplasia), assigning 2 points each to a total score ranging from 0 to 8 . The grading system was based on the sum of both scores, reaching a final total score ranging from 0 to 60 (Fortuna et al., 2013b).

\subsection{Statistical analysis}

Descriptive statistics of sociodemographic characteristics of DEB patients and controls were calculated as mean \pm standard deviation, frequency, and medians with interquartile range. Student's $t$-test were employed for calculation of means, Mann-Whitney $U$-test for medians, and $\chi^{2}$ test for gender frequency. Linear correlations were measured by the Spearman rank correlation. 
Correlations were measured between oral health-related quality of life and general quality of life with anxiety and depression, oral health-related quality of life with general quality of life, and between oral-pharyngeal disease severity and number of oral-pharyngeal sites involved. P-values were considered significant if $0.01<\mathrm{p} \leq 0.05$ and highly significant if $\mathrm{p} \leq 0.01$.

\section{Results}

3.1 Patients' sociodemographic characteristics and assessment of oral health-related quality of life and general quality of life

We enrolled 28 DEB patients ( 9 males and 19 females) and 26 control patients ( 9 males and 17 females) with a mean age of $31.0 \pm 11.4$ years and $30.4 \pm 9.6$ years, respectively. No statistical differences in age $(p=0.801)$, gender $(p=0.847)$, anxiety symptoms $(p=0.576)$ or depressive symptoms $(p=0.202)$ were found between the two groups. However, there was a significant difference in the OHIP-49 total score $(\mathrm{p}<0.001)$ (Table 1$)$.

Table 1 Demographic and clinical characteristics of Dystrophic EB and control patients

\begin{tabular}{|c|c|c|c|}
\hline & DEB patients & Control patients & \\
\hline Demographic variables & Mean $\mathbf{\text { SD }}$ & Mean \pm SD & P-value \\
\hline Age & $31.0 \pm 11.4$ & $30.3 \pm 9.6$ & 0.801 \\
\hline & Frequency & Frequency & \\
\hline Gender (Male:Female) & $9: 19$ & $9: 17$ & 0.847 \\
\hline $\begin{array}{l}\text { DEB Type } \\
\text { (Dominant: Recessive) }\end{array}$ & $8: 20$ & ----- & \\
\hline & & & \\
\hline Clinical parameters & Median (IQR) & Median (IQR) & P-value \\
\hline HAM-D & $3.5(0.5-8.0)$ & $3.5(0.7-9.0)$ & 0.576 \\
\hline HAM-A & $10.0(2.3-15.8)$ & $4.5(2.0-10.3)$ & 0.202 \\
\hline OHIP-49 (Total score) & $55.5(25.5-81.0)$ & $16.0(6.8-24.5)$ & $<0.001^{* *}$ \\
\hline
\end{tabular}

DEB, Dystrophic Epidermolysis bullosa; HAM-A, Hamilton Rating scale for Anxiety; HAM-D, Hamilton Rating scale for Depression; OHIP, Oral Health Impact Profile; SF, Short form; IQR, Interquartile range; SD, Standard Deviation.

Significant $.01<\mathrm{p} \leq .05,{ }^{* *}$ Highly Significant $\mathrm{p} \leq .01$.

The analysis of each single items of the oral health-related quality of life showed that DEB patients suffer from a higher impairment in all 7 dimensions: functional limitations $(p<0.001)$, physical pain $(\mathrm{p}=0.001)$, psychological discomfort $(\mathrm{p}=0.006)$, physical disability $(\mathrm{p}<0.001)$, psychological disability $(p=0.008)$, social disability $(p=0.004)$, and handicap $(p=0.001)$ (Table 2$)$ (Figure 2$)$.

Concerning the general quality of life, 4 out of 8 dimensions of the RAND SF-36 showed a statistical difference: physical functioning $(p<0.001)$, role limitation due to physical health $(p=0.043)$, pain $(p=0.01)$, and general health $(p=0.001)$ (Table 2) (Figure 3). 
Giulio Fortuna, Massimo Aria, Sarah Whitmire et al. / American Journal of Oral Medicine

(2016) Vol. 2 No. 2 pp. 43-59

Table 2 Oral health-related quality of life and general quality of life in DEB patients versus control group.

\begin{tabular}{|l|c|c|c|}
\hline \multicolumn{1}{|c|}{ ITEMS } & DEB patients & Control patients & \\
\hline OHIP-49 & Median (IQR) & Median (IQR) & P-value \\
\hline Functional Limitation & $13(10.3-20)$ & $7.0(2.0-9.0)$ & $<0.001^{* *}$ \\
\hline Physical Pain & $13.5(6.0-18.8)$ & $5.0(1.8-10.0)$ & $0.001^{* *}$ \\
\hline Psychological Discomfort & $8.0(2.0-11.8)$ & $1.5(0.0-5.3)$ & $0.006^{* *}$ \\
\hline Physical Disability & $11.5(4.0-15.0)$ & $0.0(0.0-2.3)$ & $<0.001^{* *}$ \\
\hline Psychological Disability & $4.0(0.0-8.0)$ & $0.0(0.0-1.0)$ & $0.008^{* *}$ \\
\hline Social Disability & $0.0(0.0-4.8)$ & $0.0(0.0-0.0)$ & $0.004^{* *}$ \\
\hline Handicap & $2.0(0.0-7.0)$ & $0.0(0.0-0.3)$ & $0.001^{* *}$ \\
\hline & & & \\
\hline SF-36 & Median (IQR) & Median (IQR) & P-value \\
\hline Physical Functioning & $80.0(46.3-93.8)$ & $100.0(90.0-100.0)$ & $<0.001^{* *}$ \\
\hline $\begin{array}{l}\text { Role limitation due to physical } \\
\text { health }\end{array}$ & $100.0(50.0-100.0)$ & $100.0(100.0-100.0)$ & $0.043^{*}$ \\
\hline $\begin{array}{l}\text { Role limitations } \\
\text { emotional problems }\end{array}$ & $100.0(8.3-100.0)$ & $100.0(66.7-100.0)$ & 0.538 \\
\hline Energy/Fatigue & & & \\
\hline Emotional well-being & $62.3(45.0-80.0)$ & $75.0(50.0-80.0)$ & 0.226 \\
\hline Social functioning & $87.5(62.5-100.0)$ & $100.0(75.5-100.0)$ & 0.167 \\
\hline Pain & $72.5(46.3-87.5)$ & $90.0(67.5-100.0)$ & $0.010^{*}$ \\
\hline General Health & $47.5(36.3-71.3)$ & $80.0(62.5-90.0)$ & $0.001^{* *}$ \\
\hline
\end{tabular}

DEB, Dystrophic Epidermolysis bullosa OHIP, Oral Health Impact Profile SF, Short form 36 IQR, Interquartile range.

Significant $.01<\mathrm{p} \leq .05,{ }^{* *}$ Highly Significant $\mathrm{p} \leq .01$. 
Giulio Fortuna, Massimo Aria, Sarah Whitmire et al. / American Journal of Oral Medicine

(2016) Vol. 2 No. 2 pp. 43-59
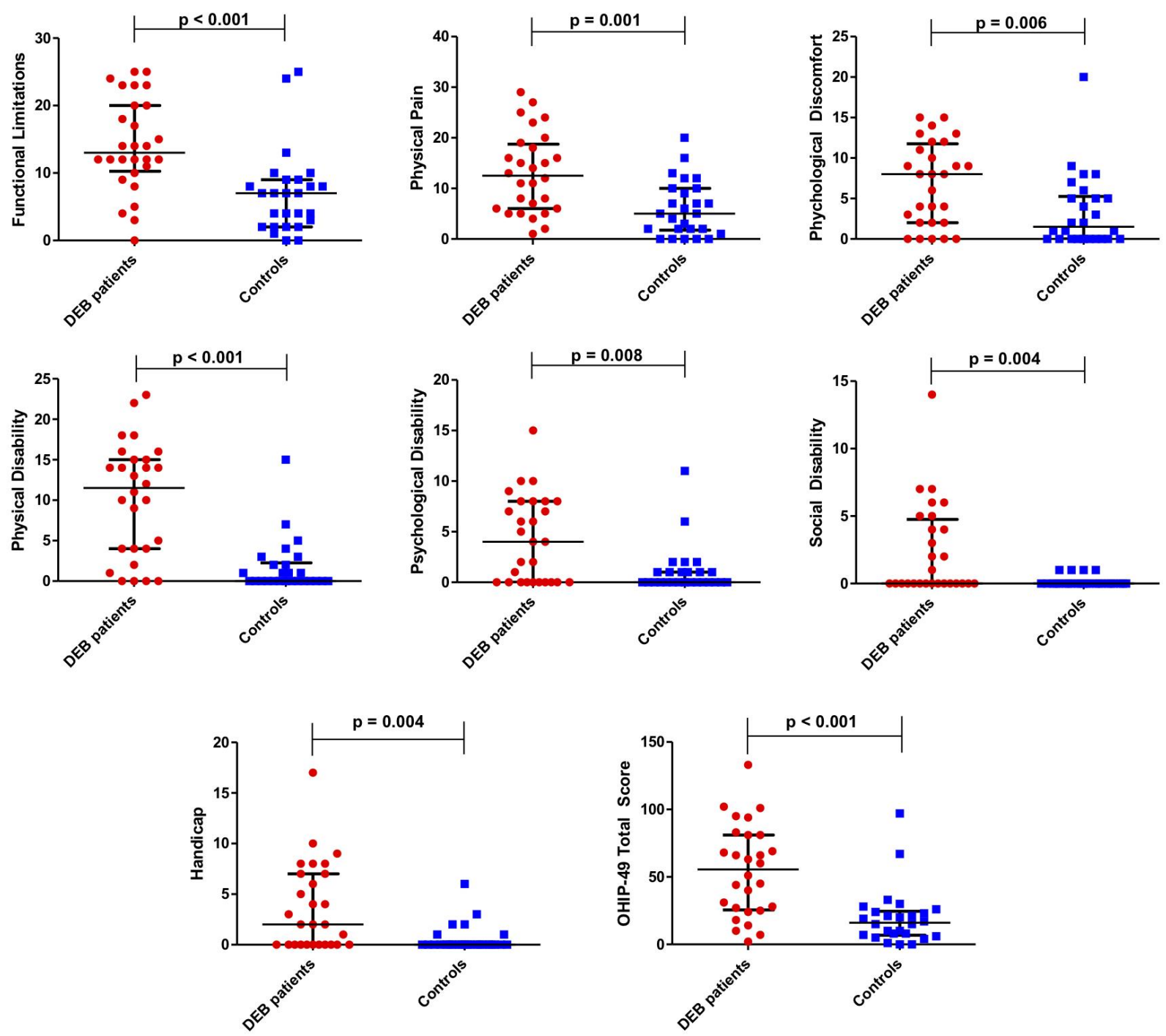

Fig. 2. Oral health-related quality of life assessment.

The Oral Health Impact Profile (OHIP-49). Dot plot of score between DEB patients and control with higher scores being indicative of a poorer oral health-related quality of life. For all graphs, center bar represents median and upper and lower bars are the $25^{\text {th }}$ and $75^{\text {th }}$ percentiles. Data were analyzed with Mann-Whitney $U$-tests. 
Giulio Fortuna, Massimo Aria, Sarah Whitmire et al. / American Journal of Oral Medicine

(2016) Vol. 2 No. 2 pp. 43-59
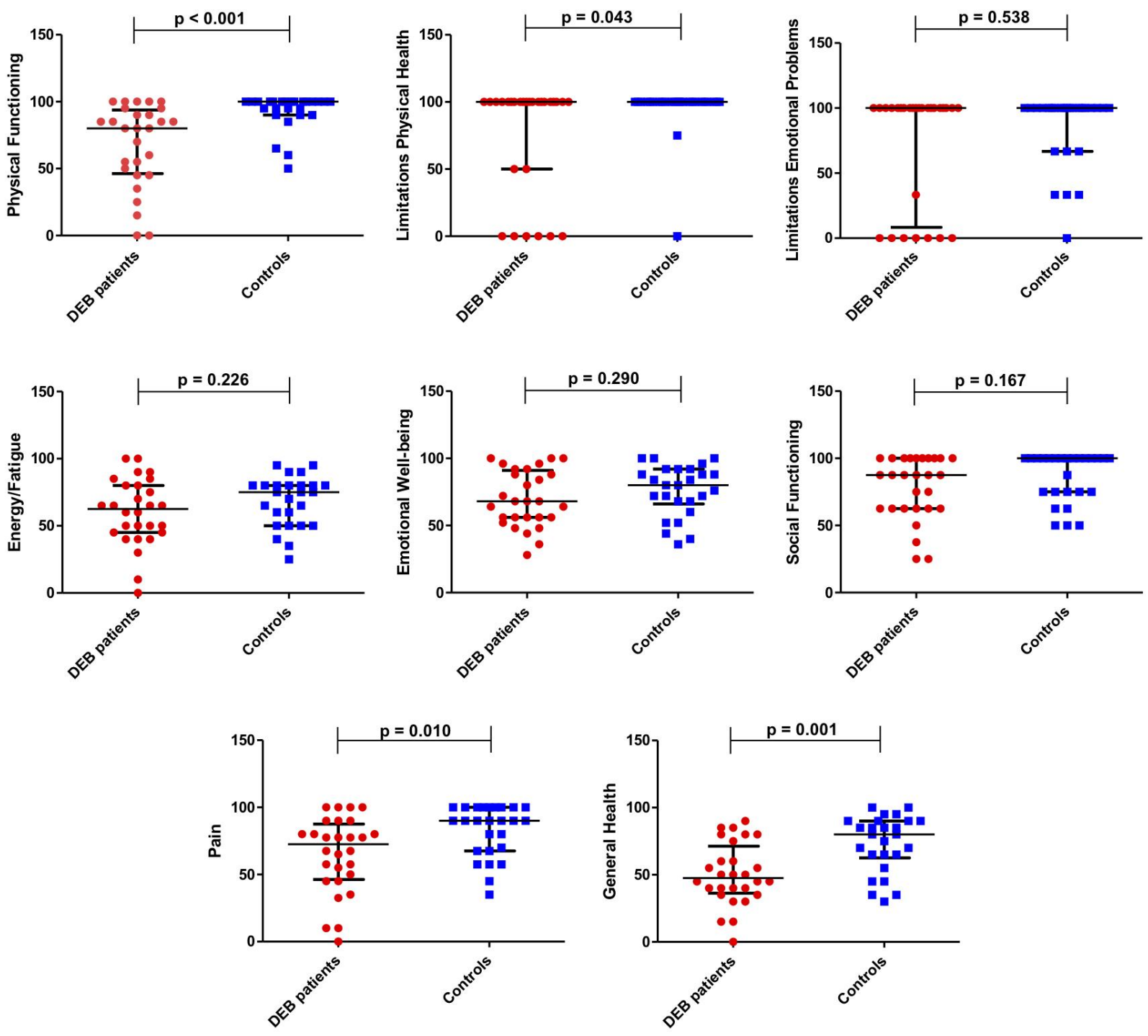

Fig. 3. General quality of Life Assessments.

RAND Short Form-36 (SF-36) Dot plot of score between DEB patients and control with higher scores being indicative of a better general quality of life. For all graphs, center bar represents median and upper and lower bars are the $25^{\text {th }}$ and $75^{\text {th }}$ percentiles. Data were analyzed with MannWhitney $U$-tests.

The radial diagrams representing all subscales of the OHIP-49 and RAND SF-36 better highlight the differences in the amount of statistically significant items between the oral health-related quality of life and the general quality of life measured in both groups (Figure 4). 

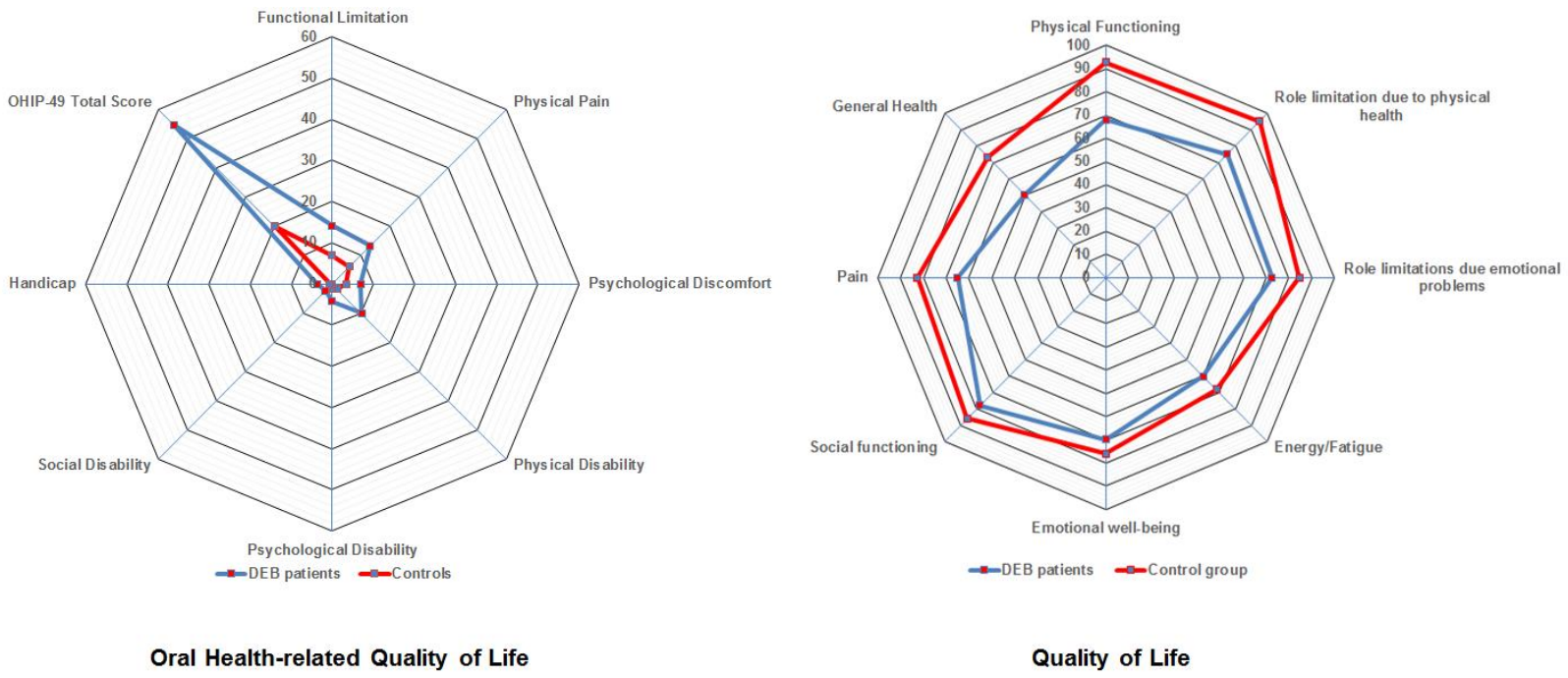

Fig. 4. Radial representation of the means (colored solid squares) of the 8 sub-dimensions assessed with the RAND SF-36 and 7 sub-dimensions plus total score assessed with the OHIP-49 between patients with DEB (solid blue line) and control patients (solid red line).

3.2 Correlation between oral health-related quality of life, general quality of life with anxiety and depression.

In both groups, the OHIP-49 total score was positively, although weakly, correlated with anxiety (p $=0.005$ and $\mathrm{p}=0.003$ in DEB patients and controls, respectively) and depressive $(\mathrm{p}=0.045$ and $\mathrm{p}=$ 0.001 in DEB patients and controls, respectively) symptoms (Table 3).

In DEB patients, a negative but not statistically significant trend ( $p>0.05$ ) was found between anxiety and depression with all dimensions of SF-36 but the role limitation due to physical health, and general health with anxiety (Table 3).

Conversely, in control patients, a negative correlation was found between anxiety and depression with physical functioning, energy/fatigue, emotional well-being, social functioning, and pain ( $\mathrm{p}<$ 0.05). In addition, a negative correlation was also found between anxiety and general health $(p=0.006)$. No correlations were found between depression and general health, or anxiety and depression with the role limitation due to physical health and the role limitation due to emotional problems ( $p>0.05$ ) (Table 3). 
Giulio Fortuna, Massimo Aria, Sarah Whitmire et al. / American Journal of Oral Medicine

(2016) Vol. 2 No. 2 pp. 43-59

Table 3 Correlation between DEB patients' oral health-related quality of life and general quality of life with psychological battery scales for anxiety and depression

\begin{tabular}{|c|c|c|c|c|c|}
\hline & \multirow[b]{2}{*}{ OHIP-49 } & \multicolumn{2}{|c|}{ HAM-A } & \multicolumn{2}{|c|}{ HAM-D } \\
\hline & & Correlation & $p$-value & Correlation & $p$-value \\
\hline $\begin{array}{l}\text { DEB } \\
\text { Patients }\end{array}$ & Total & 0.518 & $0.005^{* *}$ & 0.382 & $0.045^{*}$ \\
\hline Control & Total & 0.557 & $0.003^{* *}$ & 0.627 & $0.001^{* *}$ \\
\hline \multicolumn{2}{|r|}{ SF-36 } & Correlation & $p$-value & Correlation & $p$-value \\
\hline \multirow{8}{*}{$\begin{array}{l}\text { DEB } \\
\text { Patients }\end{array}$} & Physical Functioning & -0.256 & 0.188 & -0.124 & 0.530 \\
\hline & $\begin{array}{l}\text { Role limitation due to } \\
\text { physical health }\end{array}$ & 0.093 & 0.639 & 0.225 & 0.249 \\
\hline & $\begin{array}{l}\text { Role limitations due } \\
\text { emotional problems }\end{array}$ & -0.104 & 0.600 & -0.067 & 0.736 \\
\hline & Energy/Fatigue & -0.133 & 0.500 & -0.034 & 0.865 \\
\hline & Emotional well-being & -0.189 & 0.336 & -0.127 & 0.518 \\
\hline & Social functioning & -0.107 & 0.589 & -0.222 & 0.256 \\
\hline & Pain & -0.269 & 0.166 & -0.026 & 0.894 \\
\hline & General Health & -0.139 & 0.480 & 0.111 & 0.574 \\
\hline \multirow[t]{8}{*}{ Control } & Physical Functioning & -0.388 & $0.050^{*}$ & -0.507 & $0.008^{* *}$ \\
\hline & $\begin{array}{l}\text { Role limitation due to } \\
\text { physical health }\end{array}$ & -0.302 & 0.134 & -0.121 & 0.557 \\
\hline & $\begin{array}{l}\text { Role limitations due } \\
\text { emotional problems }\end{array}$ & -0.290 & 0.151 & $-0.450^{*}$ & $0.021^{*}$ \\
\hline & Energy/Fatigue & -0.663 & $<0.001^{* *}$ & -0.539 & $0.004^{* *}$ \\
\hline & Emotional well-being & -0.559 & $0.003^{* *}$ & -0.606 & $0.001^{* *}$ \\
\hline & Social functioning & $-0.462^{*}$ & $0.017^{*}$ & -0.568 & $0.002^{* *}$ \\
\hline & Pain & -0.527 & $0.006^{* *}$ & $-0.464^{*}$ & $0.017^{*}$ \\
\hline & General Health & -0.522 & $0.006^{* *}$ & -0.386 & 0.052 \\
\hline
\end{tabular}

DEB, Dystrophic Epidermolysis bullosa; HAM-A, Hamilton Rating scale for Anxiety; HAM-D, Hamilton Rating scale for Depression; OHIP, Oral Health Impact Profile; SF, Short form. Significant .01<p $\leq .05$, ** Highly Significant $\mathrm{p} \leq .01$.

3.3 Correlation between oral health-related quality of life and general quality of life, oral-pharyngeal disease severity score, oral-pharyngeal sites involved, and between DEB patient with dominant versus recessive form.

The assessment of oral-pharyngeal severity with the use of the EBOS failed to demonstrate any correlation with the oral health-related quality of life $(p=0.107)$. In addition, there was no correlation between the oral health-related quality of life and the number of oral-pharyngeal sites involved with oral lesions $(\mathrm{p}=0.414)$ (Table 4).

Interestingly, a negative but not statistically significant trend ( $\mathrm{p}>0.05)$ was found between total OHIP-49 and all dimensions of RAND SF-36, but the role limitation due to physical health and the role limitation due to emotional problems (Table 4). 
Giulio Fortuna, Massimo Aria, Sarah Whitmire et al. / American Journal of Oral Medicine

(2016) Vol. 2 No. 2 pp. 43-59

Table 4 Correlation between DEB patients' oral health-related quality of life with severity score, number of oral-pharyngeal sites involved and quality of life.

\begin{tabular}{|l|c|c|}
\hline & \multicolumn{2}{|c|}{ OHIP-49 } \\
\hline EBOS & Correlation & $\boldsymbol{p}$-value \\
\hline Oral-pharyngeal sites involved & 0.362 & 0.107 \\
\hline SF-36 & 0.193 & 0.414 \\
\hline Physical Functioning & & \\
\hline Role limitation due to physical health & -0.061 & 0.756 \\
\hline Role limitations due emotional problems & 0.219 & 0.263 \\
\hline Energy/Fatigue & 0.103 & 0.604 \\
\hline Emotional well-being & -0.301 & 0.120 \\
\hline Social functioning & -0.235 & 0.229 \\
\hline Pain & -0.118 & 0.551 \\
\hline General Health & -0.120 & 0.543 \\
\hline
\end{tabular}

DEB, Dystrophic Epidermolysis bullosa; EBOS, Epidermolysis bullosa oropharyngeal severity score; OHIP, Oral Health Impact Profile; SF, Short form.

Significant $.01<\mathrm{p} \leq .05,{ }^{* *}$ Highly Significant $\mathrm{p} \leq .01$.

Last, when each item of the oral health-related quality of life was analyzed dividing DEB patients in dominant and recessive, no correlations were found ( $p>0.05)$ (Table 5).

Table 5 Correlation between oral health-related quality of life and DEB type (dominant vs recessive).

\begin{tabular}{|l|c|c|c|}
\hline \multicolumn{1}{|c|}{ ITEMS } & DDEB & RDEB & \\
\hline OHIP - 49 & Median (IQR) & Median (IQR) & $\boldsymbol{p}$-value \\
\hline Functional Limitation & $10.5(4.3-14.3)$ & $14.0(12.0-22.3)$ & 0.086 \\
\hline Physical Pain & $12.0(2.8-15.5)$ & $13.5(6.3-19.8)$ & 0.285 \\
\hline Psychological Discomfort & $2.0(0.0-9.3)$ & $8.5(4.0-12.0)$ & 0.087 \\
\hline Physical Disability & $7.0(0.0-17.0)$ & $11.5(4.3-15.0)$ & 0.429 \\
\hline Psychological Disability & $0.5(0.0-5.5)$ & $5.5(0.0-8.0)$ & 0.201 \\
\hline Social Disability & $0.0(0.0-3.8)$ & $0.5(0.0-5.0)$ & 0.580 \\
\hline Handicap & $0.5(0.0-7.5)$ & $2.5(0.0-7.0)$ & 0.672 \\
\hline
\end{tabular}

DDEB, Dominant Dystrophic Epidermolysis bullosa; RDEB, Recessive Dystrophic Epidermolysis bullosa; OHIP, Oral Health Impact Profile Significant $.01<\mathrm{p} \leq .05,{ }^{* *}$ Highly Significant $\mathrm{p} \leq .01$.

\section{Discussion}

Epidermolysis bullosa still remains an incurable disease with devastating phenotypes in many subtypes. Therefore, it is not surprising to hypothesize that EB patients may develop a poor general quality of life associated with psychological impairment since childhood. Several studies have investigated this aspect and concluded that general quality of life in EB patients can be poor (Horn 
et al., 2002; Tabolli et al., 2009; Jeon et al., 2016; Angelis et al., 2016), but data on psychological aspects of EB have been conflicting. Some have reported no manifestation of anxiety and/or depressive symptoms (Fortuna et al., 2016) or other psychiatric disorders (Andreoli et al., 2002), whereas others have found a high prevalence of psychosocial problems and psychiatric symptoms (Margari et al., 2010).

These aspects of EB patients' lives have been explored in several investigations, mostly related to their cutaneous involvement; however, there is a lack of data on their oral health, which is an integral part of general health and well-being. The RAND SF-36 has a lack of disease-specific items; therefore, our study shows the advantage of combining the generic questionnaire on general health, the RAND SF-36, already utilized in EB populations (Tabolli et al., 2009), with the specific one related to the oral health, the OHIP-49, that has been validated and reliable in other oral-pharyngeal diseases (Lopez-Jornet et al., 2010; Barrios et al., 2015; Rimal et al., 2015), but has never been tested in patients with EB. The use of a generic and specific quality of life instrument has been suggested as the best option to better address the ongoing debate about whether generic or disease-specific instruments should be used (Streiner \& Norman, 2003).

The present study aimed to investigate upon oral health variables between DEB patients and a healthy control group and aimed to evaluate any possible correlation with general quality of life, anxiety, and /or depressive symptoms.

The key finding in our study was that DEB patients have a poorer oral health-related quality of life than healthy individuals in all three major dimensions: physical, psychological, and social. Indeed, upon comparison of oral health-related quality of life items, individuals belonging to the DEB group reported considerably poorer functional limitation and physical disability $(\mathrm{p}<0.001)$, followed by physical pain ( $p=0.001$ ) than healthy individuals (Figure 1). Such results were consistent with those ones from the RAND SF-36, where physical health dimension - specifically physical functional $(\mathrm{p}<0.001)$, general health $(\mathrm{p}=0.001)$, and pain $(\mathrm{p}=0.01)$ (Figure 2$)$ - was much lower than healthy control, indicating a poorer general quality of life.

Interestingly, the psychological aspect on OHIP-49 was less affected than the physical aspect, while the mental health status on the RAND SF-37 was not affected. These results seem to be in line with previous studies that showed no anxiety and/or depressive symptoms in DEB patients (Fortuna et al., 2016).

However, no significant correlations were found between the comparisons of the total OHIP-49 score with each single dimension of the RAND SF-36, or with disease severity and number of oralpharyngeal sites involved (Table 4). General health conditions and oral-pharyngeal disease severity might not have a direct impact on the oral health. Other factors, such as domiciliary oral hygiene, eating, drinking, or even speaking, might play a predominant role in the health status of the oral cavity because these daily activities can be daunting and difficult due to a severe pain associated with them.

On the other hand, the OHIP-49 total score was positively correlated with anxiety and depressive symptoms (Table 3) in both groups. This might suggest that oral health is related to psychological asset to some extent. Unfortunately, due to the cross-sectional design of the study, we were unable to address the specific causal relationship, which still remains unknown. 
Surprisingly, no correlation was found between general quality of life and anxiety/depression in DEB patients, whereas a negative correlation was found between general quality of life and anxiety and depression in the control group (Table 3). This might imply that factors other than psychological status could be contributing in their poor general quality of life. Those factors, whether sociodemographic, biological, economical, or cultural, were not established within this investigation, as it was beyond the scope of the study.

Interestingly enough, all items but role of limitations due to physical health showed negative correlation coefficients that were not significant (Table 3). However, we may hypothesize that there could be a negative trend between general quality of life and anxiety/depression in DEB patients, but a larger sample is necessary in order to prove this hypothesis.

It is important to highlight that all these questionnaires are not designed to diagnose any oralpharyngeal, systemic, or psychological diseases, as they do not contain diagnostic criteria, but to explore how well DEB patients had been doing with their general and oral health and any possible anxious-depressive symptoms associated with them. Additionally, they are not objective measures but represent subjective perceptions of human beings, a measurement of human behaviors.

Last, a lack of significant difference of oral health-related quality of life was observed between DEB patients with dominant versus patients with recessive form, which is why both groups were combined for analyses.

We recognize that our study has some limitations, including a relatively small sample size. This can be due to the nature of the disease and patient compliance. We need to acknowledge that EB is a rare disease and for some, mostly for those ones affected by RDEB, filling a questionnaire may be too exhausting. Other limitations may include the restriction to patients with dystrophic EB and using a single center, where all DEB patients were recruited from Northern Mexico. Therefore, we recommend the use of multi-centers for prospective studies - ideally with larger samples - in a variety of geographic locations in order to confirm our results.

Furthermore, a correlation between oral health-related quality of life via OHIP-49 and oral hygiene indices, caries indices, and periodontal indices was not measured. At the time of the study, this was considered too burdensome for the DEB patients; however, we intend conduct future studies to measure this. Lastly, it is important to underline that both instruments, the RAND SF-36 and the OHIP-49, could not be so sensitive, as no DEB patient was interviewed in their development.

Despite the limitations, our results emphasize the importance of the oral health in DEB patients, as this can be highly impaired, thus requiring special attention by all health-care professionals involved in the management of EB patients.

Oral cavity involvement, although less predominant than cutaneous involvement, plays a pivotal role in the global assessment of EB patients' life, due to the significant impact on their overall general health. This knowledge may contribute to developing preventive strategies and an individualized tailored treatment plan, as a part of their overall management, based on the individual EB patients' oral problems.

Therefore, even though the oral health does not significantly correlate with general quality of life of 
DEB patients in this study, this aspect should be considered in future studies on health-related quality of life in EB population.

We may conclude that in DEB patients: i) oral health-related quality of life is poorer than healthy individuals; ii) oral health-related quality of life is only affected in some dimensions; iii) oral healthrelated quality of life was correlated with anxious and depressive symptoms, but not with general quality of life; iv) no correlation was found between oral health-related quality of life with general quality of life, number of oral-pharyngeal sites involved, and oral-pharyngeal disease severity; v) no difference in oral health-related quality of life was seen between patients with dominant versus recessive form.

\section{Acknowledgments}

We are very grateful to all DEB patients for their courage, strength, and love. We would like to thank all D.eb.RA Mexico, Monterrey. A special thank you to Lic. Erika Salas for her valuable and priceless assistance.

\section{Conflict of Interest}

None

\section{Funding}

None

\section{References}

Alonso, J., Prieto, L. \& Anto, J. M. (1995). [The Spanish version of the SF-36 Health Survey (the SF-36 health questionnaire): an instrument for measuring clinical results]. Medicina Clinica, 104 (20), 771-776.

Andreoli, E., Mozetta, A., Angelo, C., Paradisi, M., \& Foglio Bonda, P.G. (2002). Epidermolysis bullosa. Psychological and psychosocial aspects. Dermatology and Psychosomatics, 3, 77-81. http://dx.doi.org/10.1159/000064496

Angelis, A., Kanavos, P., Lopez-Bastida, J., Linertova, R., Oliva-Moreno, J., Serrano-Aguilar, P., . . Network, B.-R. R. (2016). Social/economic costs and health-related quality of life in patients with epidermolysis bullosa in Europe. The European Journal of Health Economics, 17 Suppl 1 31-42. http://dx.doi.org/10.1007/s10198-016-0783-4

Arriens, C., Hynan, L. S., Lerman, R. H., Karp, D. R. \& Mohan, C. (2015). Placebo-controlled randomized clinical trial of fish oil's impact on fatigue, quality of life, and disease activity in Systemic Lupus Erythematosus. Nutrition Journal, 1482. http://dx.doi.org/10.1186/s12937-015-0068-2

Azrak, B., Kaevel, K., Hofmann, L., Gleissner, C. \& Willershausen, B. (2006). Dystrophic epidermolysis bullosa: oral findings and problems. Special Care in Dentistry, 26 (3), 111-115. http://dx.doi.org/10.1111/j.1754-4505.2006.tb01433.x

Barrios, R., Tsakos, G., Gil-Montoya, J. A., Montero, J. \& Bravo, M. (2015). Association between general and oral health-related quality of life in patients treated for oral cancer. Medina Oral Patologia Oral y Cirugia Bucal, 20 (6), e678-684. 


\section{Giulio Fortuna, Massimo Aria, Sarah Whitmire et al. / American Journal of Oral Medicine}

(2016) Vol. 2 No. 2 pp. 43-59

http://dx.doi.org/10.4317/medoral.20714

Castrejón-Pérez, R.C., Borges-Yá-ez, S.A., \& Irigoyen-Camacho, M.E. (2010). Validación de un instrumento para medir el efecto de la salud bucal en la calidad de vida de adultos mayores mexicanos. Revista Panamericana de Salud Publica, 27:321-9. http://dx.doi.org/10.1590/S1020-49892010000500001

De Benedittis, M., Petruzzi, M., Favia, G. \& Serpico, R. (2004). Oro-dental manifestations in Hallopeau-Siemenstype recessive dystrophic epidermolysis bullosa. Clinical and Experimental Dermatology, 29 (2), 128-132. http://dx.doi.org/10.1111/i.1365-2230.2004.01485.x

Fine, J. D., Bruckner-Tuderman, L., Eady, R. A., Bauer, E. A., Bauer, J. W., Has, C., . . Zambruno, G. (2014). Inherited epidermolysis bullosa: updated recommendations on diagnosis and classification. Journal of the American Academy of Dermatology, 70 (6), 1103-1126. http://dx.doi.org/10.1016/i.jaad.2014.01.903

Fine, J. D. \& Mellerio, J. E. (2009). Extracutaneous manifestations and complications of inherited epidermolysis bullosa: part II. Other organs. Journal of the American Academy of Dermatology, 61 (3), 387-402; quiz 403-384. http://dx.doi.org/10.1016/i.jaad.2009.03.053

Fine, J. D. \& Mellerio, J. E. (2009). Extracutaneous manifestations and complications of inherited epidermolysis bullosa: part I. Epithelial associated tissues. Journal of the American Academy of Dermatology, 61 (3), 367-384; quiz 385-366. http://dx.doi.org/10.1016/j.jaad.2009.03.052

Fortuna, G., Aria, M., Cepeda-Valdes, R., Garcia-Garcia, S. C., Moreno Trevino, M. G. \& Salas-Alanis, J. C. (2016). Role of dystrophic epidermolysis bullosa in anxiety, depression and self-esteem: A controlled crosssectional study. Journal of Dermatology, 43 (1), 70-78. http://dx.doi.org/10.1111/1346-8138.13027

Fortuna, G., Aria, M., Cepeda-Valdes, R., Pollio, A., Moreno-Trevino, M. G. \& Salas-Alanis, J. C. (2015). Clinical features of gingival lesions in patients with dystrophic epidermolysis bullosa: a cross-sectional study. Australian Dental Journal, 60 (1), 18-23. http://dx.doi.org/10.1111/adj.12264

Fortuna, G., Aria, M., Cepeda-Valdes, R. \& Salas-Alanis, J. C. (2015). Evaluation of internal consistency of the epidermolysis bullosa oropharyngeal severity score (EBOS). Acta Odontologica Scandinavica, 73 (2), 156160. http://dx.doi.org/10.3109/00016357.2014.931460

Fortuna, G., Chainani-Wu, N., Lozada-Nur, F., Aria, M., Cepeda-Valdes, R., Pollio, A., . . Salas-Alanis, J. C. (2013). Epidermolysis Bullosa Oropharyngeal Severity (EBOS) score: a multicenter development and reliability assessment. Journal of the American Academy of Dermatology, 68 (1), 83-92. http://dx.doi.org/10.1016/j.jaad.2012.04.009

Fortuna, G., Lozada-Nur, F., Pollio, A., Aria, M., Cepeda-Valdes, R., Marinkovich, M. P., . . . Salas-Alanis, J. C. (2013). Patterns of oral mucosa lesions in patients with epidermolysis bullosa: comparison and agreement between oral medicine and dermatology. Journal of Oral Pathology and Medicine, 42 (10), 733-740. http://dx.doi.org/10.1111/jop.12094

Fortuna, G., Pollio, A., Aria, M., Moreno-Trevino, M. G., Marasca, F. \& Salas-Alanis, J. C. (2014). Genotypeoropharyngeal phenotype correlation in Mexican patients with dystrophic epidermolysis bullosa. International Journal of Oral and Maxillofacial Surgery, 43 (4), 491-497. http://dx.doi.org/10.1016/i.ijom.2013.10.004

Hamilton, M. (1959). The assessment of anxiety states by rating. British Journal of Medical Psychology, 32 (1), 50-55. http://dx.doi.org/10.1111/j.2044-8341.1959.tb00467.x

Hamilton, M. (1960). A rating scale for depression. Journal of Neurology, Neurosurgery and Psychiatry, 23 5662. http://dx.doi.org/10.1136/innp.23.1.56 


\section{Giulio Fortuna, Massimo Aria, Sarah Whitmire et al. / American Journal of Oral Medicine}

(2016) Vol. 2 No. 2 pp. 43-59

Horn, H. M. \& Tidman, M. J. (2002). Quality of life in epidermolysis bullosa. Clinical and Experimental Dermatology, 27 (8), 707-710. http://dx.doi.org/10.1046/j.1365-2230.2002.01121.x

Jeon, I. K., On, H. R. \& Kim, S. C. (2016). Quality of Life and Economic Burden in Recessive Dystrophic Epidermolysis Bullosa. Annals of Dermatology, 28 (1), 6-14. http://dx.doi.org/10.5021/ad.2016.28.1.6

Laimer, M., Prodinger, C. \& Bauer, J. W. (2015). Hereditary epidermolysis bullosa. Journal der Deutschen Dermatologischen Gesellschaft, 13 (11), 1125-1133. http://dx.doi.org/10.1111/ddg.12774

Lobo, A., Chamorro, L., Luque, A., Dal-Re, R., Badia, X., Baro, E. \& Grupo de Validacion en Espanol de Escalas, P. (2002). [Validation of the Spanish versions of the Montgomery-Asberg depression and Hamilton anxiety rating scales]. Medicina Clinica, 118 (13), 493-499.

Lopez-Jornet, P. \& Camacho-Alonso, F. (2010). Quality of life in patients with oral lichen planus. Journal of Evaluation in Clinical Practice, 16 (1), 111-113. http://dx.doi.org/10.1111/j.1365-2753.2009.01124.x

Margari, F., Lecce, P. A., Santamato, W., Ventura, P., Sportelli, N., Annicchiarico, G. \& Bonifazi, E. (2010). Psychiatric symptoms and quality of life in patients affected by epidermolysis bullosa. Journal of Clinical Psychology in Medical Settings, 17 (4), 333-339. http://dx.doi.org/10.1007/s10880-010-9205-4

Matza, L. S., Morlock, R., Sexton, C., Malley, K. \& Feltner, D. (2010). Identifying HAM-A cutoffs for mild, moderate, and severe generalized anxiety disorder. International Journal of Methods in Psychiatric Research, 19 (4), 223-232. http://dx.doi.org/10.1002/mpr.323

Ramos-Brieva, J. A. \& Cordero-Villafafila, A. (1988). A new validation of the Hamilton Rating Scale for Depression. Journal of Psychiatric Research, 22 (1), 21-28. http://dx.doi.org/10.1016/0022-3956(88)90024-6

Rimal, J. \& Shrestha, A. (2015). Validation of Nepalese Oral Health Impact Profile14 and Assessment of Its Impact in Patients with Oral Submucous Fibrosis in Nepal. Journal of Nepal Health Research Council, 13 (29), 43-49.

Serrano-Martinez, M. C., Bagan, J. V., Silvestre, F. J. \& Viguer, M. T. (2003). Oral lesions in recessive dystrophic epidermolysis bullosa. Oral Diseases, 9 (5), 264-268. http://dx.doi.org/10.1034/j.1601-0825.2003.03971.x

Streiner, D., \& Norman, G. (2003). Health measurement scales: A practical guide to their development and use (3rd ed.). Oxford: Oxford University Press.

Tabolli, S., Sampogna, F., Di Pietro, C., Paradisi, A., Uras, C., Zotti, P., . . Abeni, D. (2009). Quality of life in patients with epidermolysis bullosa. British Journal of Dermatology, 161 (4), 869-877. http://dx.doi.org/10.1111/i.1365-2133.2009.09306.x

Wright, J. T. (2010). Oral manifestations in the epidermolysis bullosa spectrum. Dermatologic Clinics, 28 (1), 159-164. http://dx.doi.org/10.1016/j.det.2009.10.022

Wright, J. T., Fine, J. D. \& Johnson, L. (1993). Hereditary epidermolysis bullosa: oral manifestations and dental management. Pediatric Dentistry, 15 (4), 242-248.

Wright, J. T., Fine, J. D. \& Johnson, L. B. (1991). Oral soft tissues in hereditary epidermolysis bullosa. Oral Surgery, Oral Medicine, Oral Pathology, 71 (4), 440-446. http://dx.doi.org/10.1016/0030-4220(91)90426-D

Wright, J. T., Fine, J. D., Johnson, L. B. \& Steinmetz, T. T. (1993). Oral involvement of recessive dystrophic epidermolysis bullosa inversa. American Journal of Medical Genetics, 47 (8), 1184-1188. http://dx.doi.org/10.1002/ajmg.1320470811

Zimmerman, M., Martinez, J. H., Young, D., Chelminski, I. \& Dalrymple, K. (2013). Severity classification on the Hamilton Depression Rating Scale. Journal of Affective Disorders, 150 (2), 384-388. http://dx.doi.org/10.1016/j.jad.2013.04.028 